\title{
Phenotyping acute exacerbation of COPD: what more can we do for hospitalised patients?
}

\author{
Lai-Jian Cen ${ }^{1,2}$, Xiao-xian Zhang ${ }^{1,2}$ and Wei-Jie Guan ${ }^{1}$
}

${ }^{1}$ State Key Laboratory of Respiratory Disease, National Clinical Research Center for Respiratory Disease, Guangzhou Institute of Respiratory Health, The First Affiliated Hospital of Guangzhou Medical University, Guangzhou, China. ${ }^{2}$ These authors contributed equally.

Corresponding author: Wei-Jie Guan (battery203@163.com)

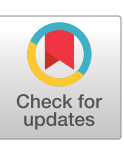

Copyright @The authors 2021

This version is distributed under the terms of the Creative Commons Attribution NonCommercial Licence 4.0. For commercial reproduction rights and permissions contact permissions@ersnet.org

Received: 28 May 2021 Accepted: 8 June 2021

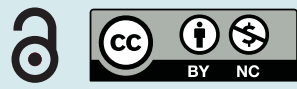

\begin{abstract}
Shareable abstract (@ERSpublications)
Hospitalised \#AECOPD are characterised by multiple facets of aetiology. The clinical interpretation of the composite phenotypes of AECOPD and the robustness of the AECOPD phenotype need to be discussed further. https://bit.ly/3grzQEO
\end{abstract}

Cite this article as: Cen L-J, Zhang X-x, Guan W-J. Phenotyping acute exacerbation of COPD: what more can we do for hospitalised patients? ERJ Open Res 2021; 7: 00362-2021 [DOI: 10.1183/ 23120541.00362-2021].

COPD is a complex and largely heterogeneous disease [1, 2]. The global prevalence of COPD is estimated to be $11.7 \%$ [3], with an estimated mortality in 2010 of around three million people [4]. The clinical management strategy has mostly been guided by the magnitude of airflow limitation, symptom burden and exacerbation risk [5]; however, these cannot fully take into account the heterogeneity of COPD. Acute exacerbations of COPD (AECOPDs) are defined as the significant worsening of respiratory symptoms which exceed the normal daily variations thus requiring changes to the current treatment [5]. AECOPD accounted for a substantial proportion of the socioeconomic burden of COPD [6], and a marked heterogeneity of the aetiology, pathophysiology and clinical manifestations of AECOPD existed [7]. In light of these heterogenous characteristics, efforts have been made to phenotype AECOPD [8]. There have been some studies focusing on the findings from clinical assessments or biomarker expression levels, highlighting the role of the exacerbation frequency [9], pathogen [10] and blood eosinophilia [11]. Nevertheless, most of these studies were restricted to the analysis of single biomarkers, some of which were not readily detectable in clinical practice, and phenotyping of AECOPD was mostly confined to the identification of infectious pathogens.

In this issue of ERJ Open Research, MACDonALD et al. [12] have explored a novel, comprehensive strategy to identify the aetiological phenotypes of AECOPD. In this prospective observational study, the phenotypes of AECOPD were identified through a constellation of metrics, ABCDEFGX, where: A=airway virus, $\mathrm{B}=$ bacterial, $\mathrm{C}=$ co-infection, $\mathrm{D}=$ depression/anxiety, $\mathrm{E}=$ =eosinophils, $\mathrm{F}=$ cardiac failure, $\mathrm{G}=$ general environment, $\mathrm{X}=$ unknown. This study has underscored a clinically relevant issue; AECOPD was comprised of multiple phenotypes. On admission, $69.2 \%$ of the 146 hospitalised patients with AECOPD had more than one phenotype of AECOPD, and only $\sim 25 \%$ of patients had a single aetiology. Importantly, these phenotypes of AECOPD could be associated with clinically meaningful outcomes. For instance, patients who experienced infective exacerbations might have a greater risk of progressing to death within 12 months after hospital discharge. During convalescence, the Hospital Anxiety and Depression Scale, blood eosinophil count and N-terminal pro-brain natriuretic peptide (NT-pro-BNP) levels remained significantly higher within the longitudinal follow-up than the levels among patients with AECOPD due to the aetiology of the D/E/F categories.

To our knowledge, this is a pioneering study that mapped the composite aetiological phenotypes of AECOPD based on the metrics that were readily derived from the routine clinical assessments. The strategy that phenotyping AECOPD based on the aetiologies might have a role in optimising the management for AECOPDs should be verified in ongoing studies. In spite of this, there remain critical issues with regard to the clinical implications of the composite aetiologies and the robustness of AECOPD phenotypes. 
The study by MacDonald et al. [12] showed that most patients might have more than one aetiology triggering the onset of AECOPD upon meticulous phenotyping. Although the relatively small sample size has hindered further analysis that fully appreciated the complexity of AECOPD. Efforts that sought to elucidate the clinical significance of the multiple facets of AECOPD are warranted.

First, patients with composite phenotypes of AECOPD might have poorer clinical outcomes. When analysing based on any single aetiology, elevated levels of cardiac biomarkers (aetiology ' $F$ ') was most common (detected in $71.2 \%$ of patients) upon the onset of AECOPD. However, patients with the infection plus the cardiac failure phenotype dominated in category ' $F$ '. This finding echoed the fact that chronic bronchial infection in COPD patients was associated with a higher incidence of cardiovascular events [13]. An unanswered question is whether infection and heart failure combined could further aggravate AECOPD compared with infection and heart failure alone. The present study revealed that co-existing infections were rather common among patients with AECOPD who had eosinophilic inflammation (16 (40\%) out of 40). Previous studies have demonstrated that the inflammatory phenotypes of COPD were associated with the sputum microbial compositions [14] and that the sputum microbial compositions could accurately predict the risk of eosinophilic exacerbation of COPD [15]. However, there also existed a dilemma that patients with AECOPD who had blood eosinophilia might benefit from corticosteroid therapy [16], which would in turn increase the risk of infections [17]. A thorough evaluation of the spectrum of the aetiology of AECOPD may help maximise the clinical benefits of individualised treatment.

Secondly, some composite aetiologies of AECOPD have also been the clinical manifestation of the comorbidities. Comorbidities have been fairly common in COPD patients and are important correlates of the poorer outcomes of AECOPD. In the present study, the pre-existing cardiovascular comorbidities might have accounted for the large proportion of category ' $\mathrm{F}$ ', although some of the COPD patients without cardiovascular disease might also have elevated NT-pro-BNP levels during exacerbations [18]. In fact, NT-pro-BNP and cardiac troponin levels were also the strong predictors of the future risks of mortality in patients with AECOPD [19, 20]. Among patients with concomitant ischaemic heart disease, the risk of cardiovascular events was markedly increased during AECOPD or at least 90 days after [21]. Apart from these, depression or anxiety has been common among patients with COPD [22]. While $24.3 \%$ of patients were identified as belonging to the aetiology category ' $\mathrm{D}$ ', depression or anxiety was present as the concurrent aetiology in this study. The results that depression or anxiety mostly occurred along with bacterial infection or cardiac failure could be partially reflected by the finding that psychiatric disorders were associated with cardiovascular comorbidities in COPD [23]. What remained less clear was whether the psychiatric disorders were more relevant to the comorbidities for COPD exacerbation. More metrics, such as obtaining a detailed medical history, might help to distinguish the pre-existing comorbidities from the trigger of AECOPD.

Finally, the robustness of the AECOPD phenotypes over time is unclear because the current assessment was conducted at a single timepoint. In the present study, a large number of patients had evidence of infections during AECOPD, and had a greater risk of having a worse prognosis despite the fact that use of antibiotics or corticosteroids had been adjusted. In fact, it would be difficult to discriminate bacterial colonisation or viral detection from pathogen infection among patients with COPD [24, 25], although new-onset infection might have a more important role in predisposing to AECOPD. Indeed, the bacterial infection, but not viral detection, was more likely to be recurrent during subsequent AECOPD episodes, as revealed in a previously study [26]. Complete longitudinally follow-ups for multiple subsequent exacerbations and recoveries should be considered for illuminating the robustness of the AECOPD phenotype. Moreover, the eosinophilic COPD exacerbations were also more repeatable during subsequent AECOPD episodes [26], and were associated with an elevated blood eosinophil count in clinically stable COPD [27]. Since the robustness of AECOPD phenotypes was related to the clinically stable states, a complete longitudinal follow-up that consisted of stable-state exacerbations and convalescence should also be planned to thoroughly illuminate the relationship of phenotypes between steady-state COPD and exacerbations.

In summary, profiling the complete spectrum of the phenotypes of AECOPD may be practical in clinical settings. More efforts should be made to unravel the clinical significance of the composite phenotypes of AECOPD on predicting the prognosis or therapeutic response, and to explore the robustness of AECOPD phenotype in follow-up studies.

Commissioned article, peer reviewed.

Conflict of interest: The authors have no conflicts relevant to the work. 
References

1 Han MK, Agusti A, Calverley PM, et al. Chronic obstructive pulmonary disease phenotypes: the future of COPD. Am J Respir Crit Care Med 2010; 182: 598-604.

2 Garudadri S, Woodruff PG. Targeting chronic obstructive pulmonary disease phenotypes, endotypes, and biomarkers. Ann Am Thorac Soc 2018; 15: Suppl. 4, S234-S238.

3 Adeloye D, Chua S, Lee $C$, et al. Global and regional estimates of COPD prevalence: systematic review and meta-analysis. J Glob Health 2015; 5: 020415.

$4 \quad$ Hoyert DL, Xu J. Deaths: preliminary data for 2011. Natl Vital Stat Rep 2012; 61: 1-51.

5 Global Initiative for Chronic Obstructive Lung Disease. Global Strategy for the Diagnosis, Management and Prevention of Chronic Obstructive Pulmonary Disease 2021 Report. https:/goldcopd.org/2021-gold-reports/

6 Guarascio AJ, Ray SM, Finch CK, et al. The clinical and economic burden of chronic obstructive pulmonary disease in the USA. Clinicoecon Outcomes Res 2013; 5: 235-245.

$7 \quad$ Ko FW, Chan KP, Hui DS, et al. Acute exacerbation of COPD. Respirology 2016; 21: 1152-1165.

8 Zhou A, Zhou Z, Zhao Y, et al. The recent advances of phenotypes in acute exacerbations of COPD. Int $J$ Chron Obstruct Pulmon Dis 2017; 12: 1009-1018.

9 Hurst JR, Vestbo J, Anzueto A, et al. Susceptibility to exacerbation in chronic obstructive pulmonary disease. N Engl J Med 2010; 363: 1128-1138.

10 Dai M-Y, Qiao J-P, Xu Y-H, et al. Respiratory infectious phenotypes in acute exacerbation of COPD: an aid to length of stay and COPD Assessment Test. Int J Chron Obstruct Pulmon Dis 2015; 10: 2257-2263.

11 Vestbo J, Vogelmeier CF, Small M, et al. Inhaled corticosteroid use by exacerbations and eosinophils: a real-world COPD population. Int J Chron Obstruct Pulmon Dis 2019; 14: 853-861.

12 MacDonald MI, Osadnik CR, Bulfin L, et al. MULTI-PHACET: multidimensional clinical phenotyping of hospitalised acute COPD exacerbations. ERJ Open Res 2021; 7: 00198-2021.

13 Martinez-Garcia M, Faner R, Oscullo G, et al. Chronic bronchial infection and incident cardiovascular events in chronic obstructive pulmonary disease patients: A long-term observational study. Respirology 2021; in press

14 Dicker AJ, Huang JTJ, Lonergan M, et al. The sputum microbiome, airway inflammation, and mortality in chronic obstructive pulmonary disease. J Allergy Clin Immunol 2021; 147: 158-167.

15 Wang J, Chai J, Sun L, et al. The sputum microbiome associated with different sub-types of AECOPD in a Chinese cohort. BMC Infect Dis 2020; 20: 610.

16 Sivapalan P, Lapperre TS, Janner J, et al. Eosinophil-guided corticosteroid therapy in patients admitted to hospital with COPD exacerbation (CORTICO-COP): a multicentre, randomised, controlled, open-label, non-inferiority trial. Lancet Respir Med 2019; 7: 699-709.

17 Sivapalan P, Ingebrigtsen TS, Rasmussen DB, et al. COPD exacerbations: the impact of long versus short courses of oral corticosteroids on mortality and pneumonia: nationwide data on 67000 patients with COPD followed for 12 months. BMJ Open Respir Res 2019; 6: e000407.

18 Adrish M, Nannaka VB, Cano EJ, et al. Significance of NT-pro-BNP in acute exacerbation of COPD patients without underlying left ventricular dysfunction. Int J Chron Obstruct Pulmon Dis 2017; 12: 1183-1189.

19 Li H, Zeng Z, Cheng J, et al. Prognostic role of NT-proBNP for in-hospital and 1-year mortality in patients with acute exacerbations of COPD. Int J Chron Obstruct Pulmon Dis 2020; 15: 57-67.

20 Pavasini R, d'Ascenzo F, Campo G, et al. Cardiac troponin elevation predicts all-cause mortality in patients with acute exacerbation of chronic obstructive pulmonary disease: Systematic review and meta-analysis. Int $J$ Cardiol 2015; 191: 187-193.

21 Kunisaki KM, Dransfield MT, Anderson JA, et al. Exacerbations of chronic obstructive pulmonary disease and cardiac events. a post hoc cohort analysis from the SUMMIT randomized clinical trial. Am J Respir Crit Care Med 2018; 198: 51-57.

22 Matte DL, Pizzichini MM, Hoepers AT, et al. Prevalence of depression in COPD: a systematic review and meta-analysis of controlled studies. Respir Med 2016; 117: 154-161.

23 Chen W, Thomas J, Sadatsafavi M, et al. Risk of cardiovascular comorbidity in patients with chronic obstructive pulmonary disease: a systematic review and meta-analysis. Lancet Respir Med 2015; 3: 631-639.

24 Leung JM, Tiew PY, Mac Aogain M, et al. The role of acute and chronic respiratory colonization and infections in the pathogenesis of COPD. Respirology 2017; 22: 634-650.

25 D’Anna SE, Balbi B, Cappello F, et al. Bacterial-viral load and the immune response in stable and exacerbated COPD: significance and therapeutic prospects. Int J Chron Obstruct Pulmon Dis 2016; 11: 445-453.

26 Mayhew D, Devos N, Lambert C, et al. Longitudinal profiling of the lung microbiome in the AERIS study demonstrates repeatability of bacterial and eosinophilic COPD exacerbations. Thorax 2018; 73: 422-430.

27 Kang HS, Kim SK, Kim YH, et al. The association between eosinophilic exacerbation and eosinophilic levels in stable COPD. BMC Pulm Med 2021; 21: 74. 